\title{
Adaptation effects of highly familiar faces: Immediate and long lasting
}

\author{
Claus-Christian Carbon \\ University of Vienna, Vienna, Austria \\ Tilo Strobach \\ Humboldt University Berlin, Berlin, Germany \\ STEPHEN R. H. LANGTON \\ University of Stirling, Stirling, Scotland \\ GÉZa HaRSÁNYI \\ Humboldt University Berlin, Berlin, Germany \\ HeLMUT Leder \\ University of Vienna, Vienna, Austria \\ AND \\ Gyula Kovács \\ University of Regensburg, Regensburg, Germany \\ and Budapest University of Technology and Economics, Budapest, Hungary
}

\begin{abstract}
A central problem of face identification is forming stable representations from entities that vary - both in a rigid and nonrigid manner - over time, under different viewing conditions, and with altering appearances. Three experiments investigated the underlying mechanism that is more flexible than has often been supposed. The experiments used highly familiar faces that were first inspected as configurally manipulated versions. When participants had to select the veridical version (known from $\mathrm{TV} / \mathrm{media} / \mathrm{movies}$ ) out of a series of gradually altered versions, their selections were biased toward the previously inspected manipulated versions. This adaptation effect ( face identity aftereffect, Leopold, Rhodes, Müller, \& Jeffery, 2005) was demonstrated even for a delay of $24 \mathrm{~h}$ between inspection and test phase. Moreover, the inspection of a specific image version of a famous person not only changed the veridicality decision of the same image, but also transferred to other images of this person as well. Thus, this adaptation effect is apparently not based on simple pictorial grounds, but appears to have a rather structural basis. Importantly, as indicated by Experiment 3, the adaptation effect was not based on a simple averaging mechanism or an episodic memory effect, but on identity-specific information.
\end{abstract}

In order to recognize a familiar face, it is a common assumption that incoming perceptual information must be matched against representations of faces stored in memory (Bruce \& Young, 1986). Theories of memory often implicitly claim that these stored representations are both stable and accurate, containing the essential information in a face that allows for its recognition (see, e.g., Bruce, 1994). However, in the present study, we show that representations of faces seem to be much more flexible and are subject to immediate adaptation following exposure to new visual information.

Human long-term memory holds information about objects, events, and affective evaluations (Bower, ThompsonSchill, \& Tulving, 1994). Representations of objects facilitate their recognition and are a prerequisite for selecting adequate actions (Baddeley, 1998). In order to recognize objects that might be viewed from different angles and under different lighting conditions, researchers have suggested that these representations comprise essential information about an object that is invariant to such changes. Faces present a particular problem for recognition since - in addition to changes in viewpoint, lighting, distance, and sizethey appear different from instance to instance because of changes in expression, hairstyle, age, and speech accompanying movements (Bruce, 1994; Leder \& Carbon, 2005). Bruce assumed that invariant structural information is abstracted from faces, allowing for their recognition despite such changes. These representations are thought to be based on the component features of the face, as well as on their

C.-C. Carbon, cce@experimental-psychology.com 
configuration (Carbon \& Leder, 2005b, 2006b; Collishaw \& Hole, 2000; Leder \& Bruce, 2000; Leder \& Carbon, 2006; Mondloch, Le Grand, \& Maurer, 2002). Moreover, a number of theories claim that face recognition occurs by reference to a prototype (Benson \& Perrett, 1993). For example, some authors (Goldstein \& Chance, 1980; Valentine $\&$ Bruce, 1986a, 1986b) have suggested that faces may be encoded by reference to this prototype or to a schematic representation, which emerges as a result of a person's experience with faces over a lifetime (Schwaninger, Carbon, \& Leder, 2003).

Researchers suppose that such a prototype is unlikely to be modified in the course of single incidents (Bruce, Doyle, Dench, \& Burton, 1991). Nevertheless, such a prototype has to be flexible enough to integrate new information that might help one to recognize the most recent appearance of the face. For example, recognizing familiar faces in spite of short-term or long-term changes is probably most efficiently done by integrating these changes into the representation of that person's face. Such an integrating mechanism should also apply to ongoing long-term changes of a face during a human's life span, especially the shifting of the facial configuration from a baby face to a matured appearance. However, such a mechanism should also be capable of integrating short-term changes. For example, the current mental and physical status may alter the facial appearance as much as would hairstyles and make-up. Without a rather flexible mechanism that integrates these types of changes, perceivers' recognition performances would be suboptimal. From an evolutionary point of view, this would be a functional disadvantage due to lacking adaptation. On the other hand, it is also important that (face) representations are sufficiently stable and rigid to allow for reliable recognition. If representations are adapting too rigorously toward recently perceived information, such an over-adaptive mechanism could also be disastrous for a recognition system. Thus, there must be a clever balance between both poles of stability and flexibility.

To challenge this adaptation mechanism, the present experiments used highly familiar faces, which are per definition highly stable in some respect, but should also be flexible enough to be updated. Up to now, most face adaptation effects were demonstrated for unfamiliar faces (see, e.g., Webster \& MacLin, 1999), but only a few were demonstrated for highly familiar faces (see Carbon \& Leder, 2005a, 2006a). Judgments concerning the veridicality of highly familiar faces, whose representations are thought to be highly reliable (Bruce, Henderson, Newman, \& Burton, 2001), were strongly influenced by recent visual inputs (Carbon \& Leder, 2005a).

\section{The Present Study}

In the present study, we examined the quality and durability of such an adaptation mechanism (in the sense of a face identity aftereffect, see Leopold et al., 2005) by determining the recognition performance of familiar faces after having inspected deviated versions of these faces. Two major types of information were varied in the present experiments to reveal the quality and durability of the underlying mechanism: (1) the delay between the adaptation (inspection) phase and the test phase, from $5 \min$ to $24 \mathrm{~h}$, and (2) the overlap of information between the adaptation phase and the test phase, by varying the pictorial compatibility between the stimuli of both phases.

In all of the experiments, participants first inspected versions of familiar faces, wherein the distance between face features had been greatly altered (as in Carbon \& Leder, 2005a; Carbon \& Leder, 2006a). Note that the specific types of alterations that we used here (configural changes) are not changes to faces that would commonly be encountered in real life; however, these changes can be operationalized by a clear procedure and can enable the systematic use of gradually altered versions. After the inspection phase, participants had to select in a test phase the veridical face from a series of similar faces, which were morphed within a continuum of changes. If the inspection leads to an adaptation, we would expect that participants misjudge altered versions as veridical versions - specifically that they judge those versions as veridical which are slightly altered toward the inspected version. Note that the manipulated versions do not resemble caricatures in which distinctive features are systematically expanded; those reveal representations that are recognized more easily (Benson \& Perrett, 1994; Lee, Byatt, \& Rhodes, 2000). Furthermore, we used highly familiar faces, in contrast to other studies that investigated such face adaptation effects ${ }^{1}$ using preexperimentally unfamiliar faces (e.g., Webster \& MacLin, 1999).

Using highly familiar faces as test stimuli makes an essential difference from most of the earlier studies (e.g., Webster \& MacLin, 1999), because highly familiar representations are presumably more stable and resistant to alterations because of their more elaborated and densely interconnected memory structures (Rossion, Schiltz, Robaye, Pirenne, \& Crommelinck, 2001). So far, only a few studies have investigated the adaptation of highly familiar faces, such as those of celebrities (Carbon \& Leder, 2005a) or famous portraits like Da Vinci's Mona Lisa (Carbon \& Leder, 2006a). The investigation of adaptation effects of highly familiar faces in combination with long-term delays between the inspection and test phase and a variation of information overlap between both of these phases should help one to understand what Leopold et al. (2005) had referred to as a key issue of research on adaptation effects: "Little is known about the build up and decay of the face aftereffect" (p. 897).

\section{EXPERIMENT 1 Image Specificity With a 5-min Delay}

In face adaptation research, inspected (distorted) faces and tested faces are mostly based on the same picture of the person. Moreover, adaptation effects are mainly investigated within a very short time span, ranging from milliseconds (see, e.g., Leopold, O'Toole, Vetter, \& Blanz, 2001; Rhodes et al., 2004; Webster, Kaping, Mizokami, \& Duhamel, 2004) to seconds (e.g., Carbon \& Leder, 2005a). The experiments presented herein varied the overlap of pictorial information between inspection (adaptation) phase and test phase, as well as the delay between both phases. Doing so helps one to understand the underlying mechanisms in terms of image-specificity and durability of adaptations. 


\section{Method}

Participants. Thirty-six participants were tested individually (23 females, mean age 29.9 years). Eight participants were students of psychology at Freie Universität Berlin; all other participants were students with different majors at FU Berlin.

Apparatus and Stimuli. Face pictures of celebrities from several areas of public interest (sport, politics, television, etc.) were selected as familiar faces. Two different pictures (A and B) of 27 famous individuals ${ }^{2}$ were selected as base material.

The stimuli were selected according to the following criteria: They should contain a full frontal face with high resolution; the portrayed person would be highly familiar to German students, and both pictures of one person should differ to a certain degree.

The entire set of images was divided into three subsets to create three different levels of overlapping pictorial information between images seen in the inspection phase and the test phase. These three sets included in the between-subjects factor overlap are referred to as pictorial, structural, and transfer in what follows. For the set of pictorial stimuli, the pictures of the inspection phase and the test phase were pictorially identical, whereas for the set of the structural stimuli, different images of the same person were used in the inspection and the test phase. Celebrities included in the transfer set were only presented as a single image version in the test phase. The experimental factor overlap is illustrated in Figure 1.

Each image was manipulated - resulting in 11 different versionsby changing the distance between the eyes and the mouth; this shifting technique is similar to that in other studies using configurally distorted facial material (e.g., Carbon \& Leder, 2005a; Carbon \& Leder, 2006a; Faulkner, Rhodes, Palermo, Pellicano, \& Ferguson, 2002). There were five versions for which the distances between eyes and mouth were linearly decreased by 3 pixels (minus 1 , minus 2 , minus 3 , minus 4 , and minus5), one version that was veridical (original), and five more versions for which the distances between the eyes and mouth were linearly increased by 5 pixels ( plus 1 , plus 2, plus 3 , plus 4 , and plus5). Importantly, a prestudy showed that all altered versions (from minus 5 to plus5) could still be identified as belonging to the same celebrity ("Who is this?"; $p_{\text {recognition }}>.9$ ). The size of the pictures was approximately $220 \times 240$ pixels. An example of all the versions (here with the face of Princess Diana) is shown in Figure 2.
The experiment was run on a Macintosh eMac with an integrated 17 -in. CRT $(1,024 \times 768$ pixels $)$.

Procedure. The experiment consisted of three phases. In the first phase, participants had to inspect minus 5 faces, original faces, or plus 5 faces (realized by a between-subjects design). After a delay of 5 min, participants had to perform the second phase, in which they had to select the veridical face from a sequence of face versions. In the third phase, participants had to perform both a familiarity and typicality decision task.

Phase 1: Inspection phase. In the inspection phase, the faces were all shown as minus 5 versions, original versions, or plus 5 versions in a between-subjects design. In order to let the participants inspect the faces, they were given the cover task of categorizing the faces according to gender. In order to maximize the duration of inspection, the difficulty of the task was increased by presenting the faces randomly for $1 \mathrm{sec}$ (in four out of six of all cases), $2 \mathrm{sec}$ (one out of six), or $3 \mathrm{sec}$ (one out of six) at nine random places on the screen. Across all trials, each face was shown for $81 \mathrm{sec}$ in the inspection phase altogether.

Phase 2: Test phase. After a delay of $5 \mathrm{~min}$, the test phase with faces pertaining to the pictorial, structural or transfer set followed. The images of the celebrities were randomly assigned to one of these three face sets across participants. Participants were asked to select the veridical version (test face) from a series including all 11 face versions. Note that the participants were explicitly instructed to make their decisions on the basis of their world knowledge (images known from $\mathrm{TV} /$ media/movies), but not on any experimentally seen images. By pushing the right button, the participants viewed images toward the plus 5 version; pushing the left button revealed images toward the minus 5 version. The starting image in the set was minus 1, original, or plus 1, in random order to diminish anchor effects resulting from the first appearance of the test faces. Participants were asked to use the left and right buttons to browse through the sequence of face versions in their individually selected pace and to press the space bar to select what they considered the veridical version of the face. Overall, participants had to make the veridicality selection for 3 (overlap information: pictorial, structural, transfer) $\times$ 9 (faces) $\times 3$ (start-test face: minus 1 , original, plus 1$)=81$ images. For the pictorial condition, the versions from which the participants

\section{Overlap Information}

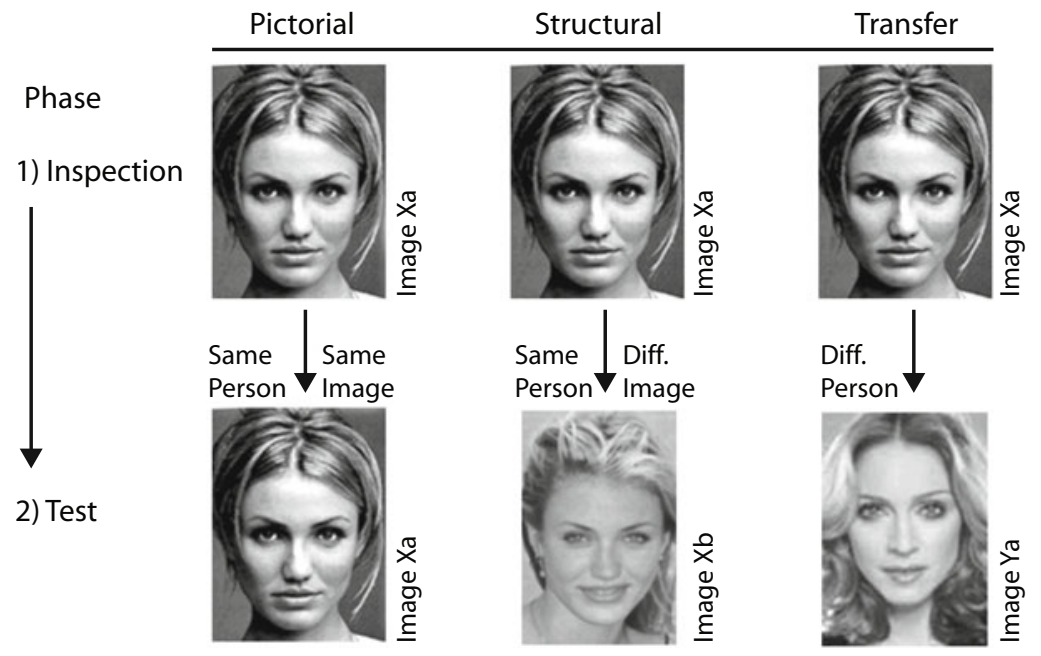

Figure 1. Illustration of the different overlap information conditions. On the left side, the pictorial condition (inspection and test phase: Cameron Diaz; same images), in the middle, the structural condition (inspection and test phase: Cameron Diaz; different images), and on the right, the transfer condition (different persons: inspection, Cameron Diaz; test phase, Madonna). 


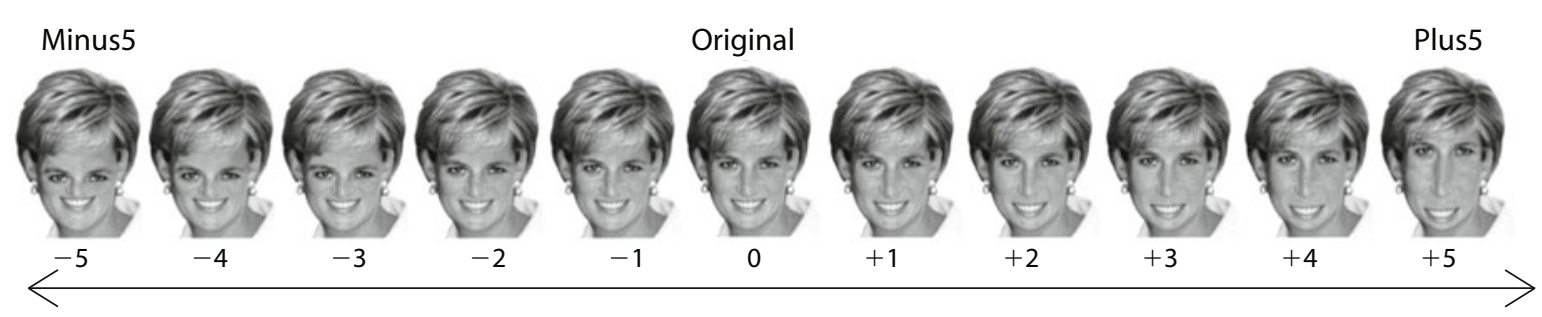

Figure 2. The range of configural manipulations illustrated by the face of Princess Diana. The scale ranges from -5 , equivalent to minus5, up to +5 , equivalent to plus5. Zero indicates the original version.

had to select the veridical one were based on the same image as that in the inspection phase. For the structural condition, different base-image versions of the same person that had been inspected in the previous inspection phase were used. For the transfer condition, entirely new images of persons other than those who were inspected during the inspection phase were used (see Figure 3 for an illustration of the trial schema of Phase 2).

Phase 3: Familiarity and typicality decision phase. At the end of the experiment, participants had to evaluate all veridical versions regarding their familiarity and typicality. This was done to ensure that they were familiar with the faces used. The faces were shown sequentially on the screen without time limits. First, the participants had to evaluate all faces within one block in terms of their familiarity (0, "unfamiliar;" 1, "familiar"), then they had to evaluate them regarding their typicality (7-point Likert scale, from 1, very untypical to 7 , very typical) on the basis of their personal evaluation.

The trials, presented in a randomized order per block, were controlled by the experimental software PsyScope PPC 1.25 (Cohen, MacWhinney, Flatt, \& Provost, 1993) run on MacOS 9.2.2. The entire procedure, including the evaluation task at the end, lasted approximately $45 \mathrm{~min}$.

\section{Results and Discussion}

On average, $94.9 \%$ of all target faces presented in the test phase were familiar to the participants. Only these familiar faces were included in the following analyses, since only these faces can have a more or less strong memory representation. The main dependent variable of interest was the selected target (test face) of familiar faces, which is an indicator of whether the prior inspection of - for instance, extremely deviated-face versions had altered the representations of familiar faces (see Table 1).

According to the experimental design, test face was analyzed by a three-way mixed-design ANOVA, with the between-subjects factor inspection face (minus5, original, plus5), and the within-subjects factors overlap (pictorial, structural, transfer) and start-test face (the initially presented test face: minus 1, original, plus 1). There was a main effect of inspection face $[F(2,33)=201.27, p<$ $\left..0001, \eta_{\mathrm{p}}^{2}=.924\right]$, with the following means: minus $5(M=$ $-1.57)$, original $(M=0.054)$, and plus $5(M=1.96)$. Bonferroni comparisons revealed significant differences between all possible pairs $(p \mathrm{~s}<.0001)$. The main effect of start-test face was also significant $[F(2,66)=49.56$, $\left.p<.0001, \eta_{\mathrm{p}}^{2}=.600\right]$, with the following means: minus 1 $(M=-0.17)$, original $(M=0.19)$, and plus1 $(M=0.42)$. Bonferroni comparisons showed significant differences between all possible pairs $(p s<.0001)$. Furthermore, there was an interaction of overlap and inspection face $\left[F(4,66)=11.71, p<.0001, \eta_{p}^{2}=.415\right]$ (see Figure 4)

Concerning the interaction between overlap and inspection face, analysis of the simple main effects of inspection face showed strong effects for all levels of overlap

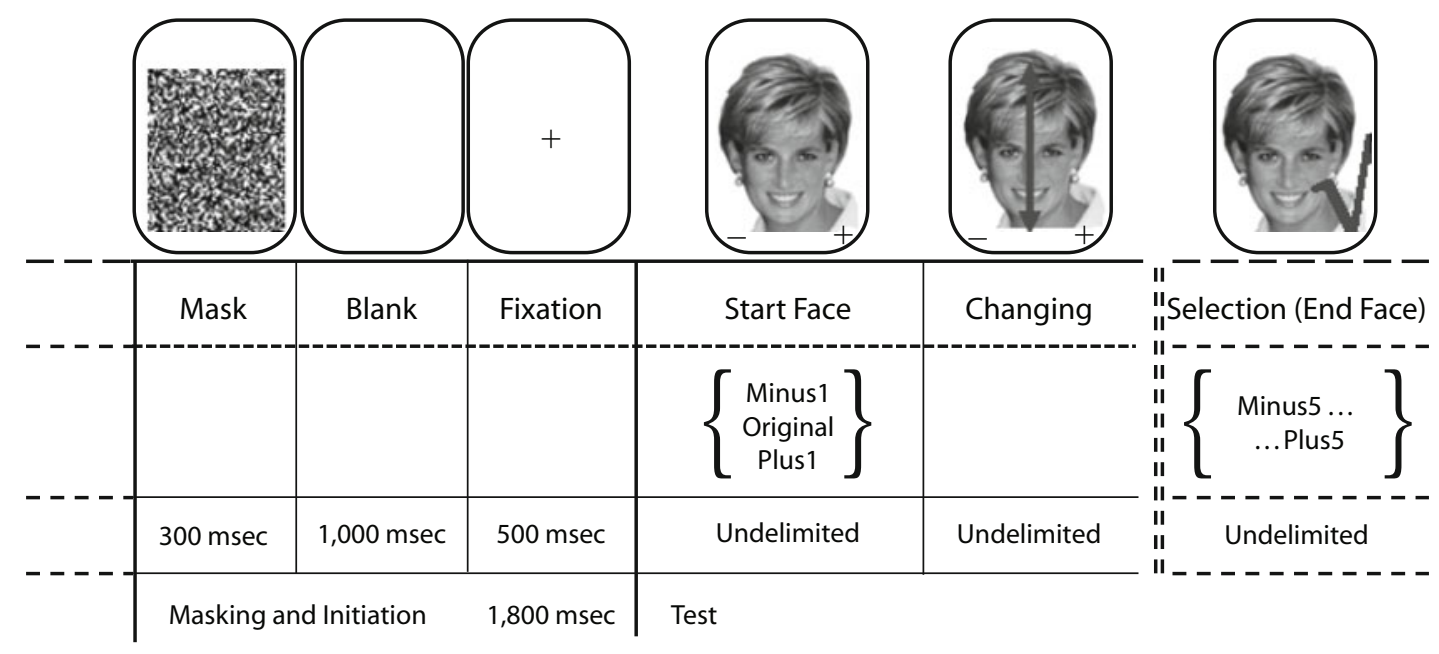

Figure 3. Time course of a trial from the test phase. Every trial started with a forward mask, followed by a blank screen and the presentation of a fixation cross. Then, the actual test trial began by starting with a randomly selected face version out of the possible versions minus1, original, and plus1. Then, the participant was able to browse the sequence of face versions until he/she selected the version that was supposed to be veridical. 
Table 1

Mean Selections and Standard Deviations As Veridical for Experiment 1

\begin{tabular}{|c|c|c|c|c|c|c|c|}
\hline \multirow[b]{3}{*}{ Overlap } & \multirow{3}{*}{$\begin{array}{l}\text { Start-Test } \\
\text { Face }\end{array}$} & \multicolumn{6}{|c|}{ Inspection Face } \\
\hline & & \multicolumn{2}{|c|}{ minus 5} & \multicolumn{2}{|c|}{ original } & \multicolumn{2}{|c|}{ plus 5} \\
\hline & & $M$ & $S D$ & $M$ & $S D$ & $M$ & $S D$ \\
\hline \multirow{3}{*}{ Pictorial } & minus 1 & -2.05 & 0.54 & -0.31 & 0.5 & 2.05 & 0 \\
\hline & & -1 & 0. & 0 & 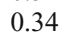 & 2.26 & 0 \\
\hline & $p$ & -1 & 0. & 0. & 0.40 & 3 & \\
\hline \multirow[t]{3}{*}{ Structural } & $m$ & -1.89 & 0.70 & -0.55 & 0.30 & 1.77 & 0. \\
\hline & & -1.74 & 0.46 & & 0. & 1.90 & \\
\hline & plus & -1 & 0.75 & 0.39 & 0.26 & 2.31 & \\
\hline \multirow[t]{3}{*}{ Transfer } & minus 1 & -1.74 & 0.74 & -0.18 & 0.36 & 1.40 & 0.7 \\
\hline & & & 0.8 & 0. & 0. & 1.71 & \\
\hline & plus1 & -1 & 0.48 & 0.31 & 0.32 & 1.89 & $0.6 \mathrm{C}$ \\
\hline
\end{tabular}

$\left[F \mathrm{~s}(2,33)>106.22, p \mathrm{~s}<.0001, \eta_{\mathrm{p}}^{2} \mathrm{~s}>.866\right]$. In order to contrast the strengths of inspection face effects on the different levels of overlap, we further analyzed the simple main effects of overlap on all three levels of inspection face. These analyses showed that there were no statistical differences between pictorial and structural faces on the minus 5 and plus 5 levels ( $p \mathrm{~s}>.118$ ), but significant differences were found between pictorial and transfer on both of these levels $(p s<.006)$. Regarding the original inspection face, there were no differences between any of these overlap levels $(p s>.99)$. Thus, pictorial and structural faces were processed in a similar way.

The analyses revealed important results on the specificity of adaptation effects. (1) Adaptation effects are not only limited to preexperimentally unfamiliar faces, but are also (even very strongly), demonstrable for familiar faces. (2) Adaptation effects seem to have some sort of representational basis and are not only perceptual effects, since the inspection and the test phase were separated by a 5-min delay. (3) Adaptation effects were most prominent when identity-specific material had been inspected before; however, adaptation effects were also quite strong toward so-called transfer faces, which had not been inspected in the experiment before. Thus, an explanation based on a more general effect of adaptation cannot be excluded. Alternatively, one must note that an episodic memory effect (Tulving, 1972) can also not be fully excluded by the present experiment. Due to the main experimental variation of inspection face realized as a betweensubjects factor, participants may have remembered the general experimental condition as such, which in turn might have altered their selections in the test phase afterwards. To address this alternative hypothesis explicitly, Experiment 3 was conducted later on.

In order to explore the quality and the duration of the adaptation effect further, we conducted Experiment 2, which was similar to Experiment 1, but used a much longer delay of $24 \mathrm{~h}$ between the inspection and test phases.

\section{EXPERIMENT 2 Image Specificity With a 24-h Delay}

Experiment 2 expanded the delay between inspection and test phase from $5 \mathrm{~min}$ to $24 \mathrm{~h}$. We were particularly interested in two experimental questions, which can be addressed by the specific experimental setting of Experiment 2: (1) Generally, does the extended delay weaken the adaptation effect? Up until now, adaptation experiments with faces were only able to find adaptations for a very short timeframe. Extending the phase between inspection and test to $24 \mathrm{~h}$ enables us to test whether the effect really depends on a more representational or perceptual basis. (2) Specifically, does the interrelation between different levels of overlap change over time? The combination of a longer delay with different levels of information overlap gives us the chance to analyze further the basis of the effect. When adaptations after such a long delay are not only limited to pictorial stimuli, but can even be demonstrated for structural stimuli, then such adaptations take place on an identity-specific basis.

\section{Method}

Participants. Thirty-six participants were tested individually (31 females, mean age 25.8 years). Twenty-two participants were students of psychology at Freie Universität Berlin; all other participants were students with other majors at FU Berlin.
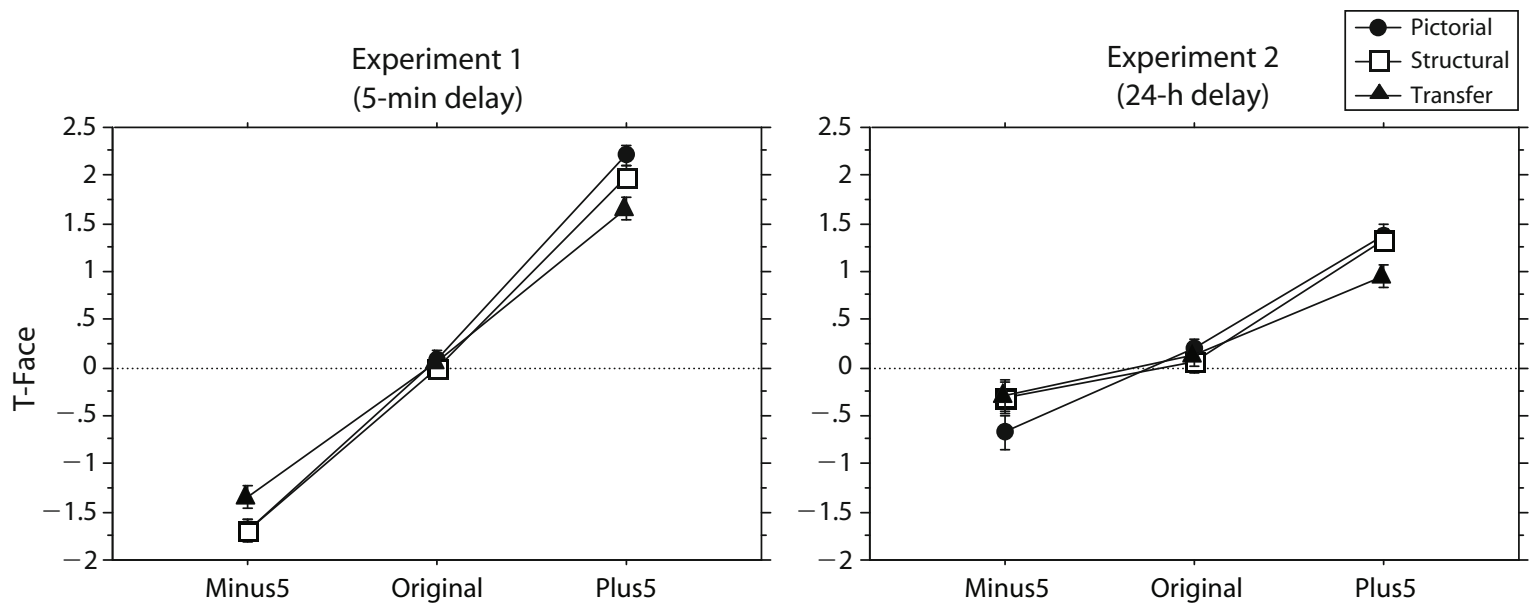

Figure 4. Mean selections as veridical for Experiments 1 and 2 as a function of inspection face and overlap (pictorial, structural, transfer). Error bars are $S E$ s. 
Apparatus and Stimuli. The apparatus and stimuli were identical to those used in Experiment 1.

Procedure. The procedure of Experiment 2 was identical to that of Experiment 1, except for the delay between the inspection and test phase. To extend this delay, the experimental procedure was interrupted after the inspection phase, and participants were invited to return the next day. Thus, the delay was extended from $5 \mathrm{~min}$ to approximately 1 day (mean delay, $24.2 \mathrm{~h}$; minimum delay, 18:05 h; maximum delay, $30: 17 \mathrm{~h}$ ).

\section{Results and Discussion}

On average, $97.0 \%$ of all target faces presented in the test phase were familiar to the participants. As for the analyses before, only data referring to these familiar faces were included in the following analyses (see mean data in Table 2).

We tested the adaptation effect by conducting a threeway mixed-design ANOVA with the between-subjects factor inspection face (minus5, original, plus5) and the within-subjects factors overlap (pictorial, structural, transfer) and start-test face (minus1, original, plus 1). As a dependent variable, we used the selected face version (test face).

There was a main effect of inspection face $[F(2,33)=$ $\left.18.12, p<.0001, \eta_{\mathrm{p}}^{2}=.523\right]$, with the following means: minus5 $(M=-0.43)$, original $(M=0.13)$, and plus $5(M=$ 1.22). Bonferroni comparisons revealed significant differences between minus 5 and plus 5 and between plus 5 and original $(p \mathrm{~s}<.0004)$, but only a trend for a difference between minus 5 and original ( $p=.0554$, n.s.). Additionally, there was a significant main effect of start-test face $\left[F(2,66)=34.90, p<.0001, \eta_{\mathrm{p}}^{2}=.514\right]$, with the following means: minus1 $(M=0.06)$, original $(M=0.31)$, and plus1 $(M=0.56)$. Bonferroni comparisons revealed significant differences between all possible pairs $(p s<.0001)$. Furthermore, there was an interaction between overlap and inspection face $\left[F(4,66)=7.27, p<.0001, \eta_{\mathrm{p}}^{2}=.306\right]$ (see Figure 4$)$. The overall resulting pattern was very similar to that of Experiment 1. We further investigated the interaction between overlap and inspection face by calculating the simple main effects of overlap. For minus 5 inspection faces, we found significant differences between pictorial and structural and between pictorial and transfer $(p \mathrm{~s}<.011)$. For plus 5, we found differences between pictorial and transfer, and between structural and transfer $(p s<.006)$.

In order to test for delay effects on the strength of the adaptation effect, we compared Experiments 1 and 2 in

Table 2

Mean Selections and Standard Deviations As Veridical for Experiment 2

\begin{tabular}{|c|c|c|c|c|c|c|c|}
\hline \multirow[b]{3}{*}{ Overlap } & \multirow{3}{*}{$\begin{array}{l}\text { Start-Test } \\
\text { Face }\end{array}$} & \multicolumn{6}{|c|}{ Inspection Face } \\
\hline & & \multicolumn{2}{|c|}{ minus 5} & \multicolumn{2}{|c|}{ original } & \multicolumn{2}{|c|}{ plus 5} \\
\hline & & $M$ & $S D$ & $M$ & $S D$ & $M$ & $S D$ \\
\hline \multirow[t]{3}{*}{ Pictorial } & minus 1 & -0.90 & 1.04 & -0.04 & 0.59 & 1.14 & 0.83 \\
\hline & original & -0.68 & 1.06 & 0.16 & 0.49 & 1.43 & 0.69 \\
\hline & plus 1 & -0.43 & 1.17 & 0.48 & 0.71 & 1.55 & 0.79 \\
\hline \multirow[t]{3}{*}{ Structural } & minus 1 & -0.67 & 0.77 & -0.30 & 0.53 & 1.21 & 0.67 \\
\hline & original & -0.15 & 1.14 & 0.12 & 0.48 & 1.33 & 0.53 \\
\hline & plus 1 & -0.10 & 1.00 & 0.33 & 0.64 & 1.47 & 0.59 \\
\hline \multirow[t]{3}{*}{ Transfer } & minus 1 & -0.66 & 0.88 & 0.17 & 0.53 & 0.90 & 0.67 \\
\hline & original & -0.29 & 0.95 & 0.03 & 0.60 & 0.82 & 0.74 \\
\hline & plus1 & 0.06 & 1.03 & 0.53 & 0.69 & 1.16 & 0.71 \\
\hline
\end{tabular}

an integral analysis. This was done in a four-way mixeddesign ANOVA with the between-subjects factors delay ( $5 \mathrm{~min}, 24 \mathrm{~h}$ ) and inspection face, and the within-subjects factors overlap and start-test face. As before, test face was used as the dependent variable.

Again, there was a large main effect of inspection face $\left[F(2,66)=124.30, p<.0001, \eta_{\mathrm{p}}^{2}=.790\right]$, with the following means: minus $5(M=-1.00)$, original $(M=0.09)$, and plus5 $(M=1.59)$. Bonferroni comparisons revealed significant differences between all possible pairs $(p s<$ $.0001)$. There was also a large main effect of start-test face $\left[F(2,132)=83.40, p<.0001, \eta_{\mathrm{p}}^{2}=.558\right]$, with the following means: minus1 $(M=-0.06)$, original $(M=$ $0.25)$, and plus1 $(M=0.49)$. Bonferroni comparisons revealed significant differences between all possible pairs $(p \mathrm{~s}<.0001)$. Furthermore, there was an interaction between overlap and inspection face $[F(4,132)=16.97, p<$ $\left..0001, \eta_{\mathrm{p}}^{2}=.340\right]$, and most importantly, an interaction between delay and inspection face $[F(2,66)=16.36, p<$ $\left..0001, \eta_{\mathrm{p}}^{2}=.331\right]$. Concerning the latter interaction, we analyzed simple main effects of delay on the different levels of inspection face. The adaptation effect after a delay of $24 \mathrm{~h}$ was weaker, as indicated by significant effects of delay on minus 5 and plus $5\left[F_{\mathrm{S}}(1,66)>9.87, p<.003\right.$, $\eta_{\mathrm{p}}^{2}>$.130]: The selected test face was less distorted in the 24-h condition than in the 5-min condition.

Thus, the analyses revealed two important results. (1) The adaptation effect was rather enduring, although it was weakened when directly compared with the 5-min delay condition. This result is in accordance with recent findings of adaptation effects with unfamiliar faces that were tested in a much more restricted temporal range (Leopold et al., 2005). However, as indicated by a significant effect of inspection face for the 24-h condition, the adaptation effect was still very prominent even after one day! (2) The quality of the adaptation effect does not appear to change over time. As indicated by a nonexisting interaction between delay and overlap, there was no modification of the adaptation effect for different inspection-test compatibilities.

Experiment 1, as well as Experiment 2, used the factor overlap in a between-subjects design regarding the main experimental factor, inspection face. According to this specific design, all inspected faces were manipulated in the same way and to the same extent. Therefore, one can neither reliably conclude that the adaptation effect is based on a general effect that changes toward the average of all experimentally seen faces, nor that the effect is based on identityspecific adaptation. As was pointed out in the discussion of Experiment 1, with a between-subjects variation of inspection time, episodic memory effects can also not be excluded as a main contribution for the adaptation effects found. Therefore, we conducted Experiment 3 with an experimental paradigm that varied the factor inspection face as a within-subjects variable. If the evaluation of veridicality of a face is based on the average of inspected faces, we would expect a zero effect of inspection face when all levels of this factor are used with the same frequency. However, if the inspection of one specifically manipulated face is related to the later evaluation of the facial veridicality of this specific person, then we would expect strong effects of inspection 
face. The same rationale can be used for investigating potential episodic memory effects: In the between-subjects designs of Experiments 1 and 2, participants might have memorized the episode of seeing (for instance) extremely compressed faces in general. Thus, their evaluation in the test phase might have been biased toward episodic memory traces. When using a within-subjects variation of inspection face, there is not only one single episode but three, since participants see minus5, original, and plus 5 faces.

\section{EXPERIMENT 3 Image-Specific Versus Prototype Effects}

Experiment 3 used a delay paradigm just like that in Experiment 1, in which the inspection and test phase were separated by a 5-min break. In contrast to Experiments 1 and 2 , in which the factor inspection face was used in a between-subjects manner, Experiment 3 varied inspection face within participants in order to test whether the adaptation effect is based on more general averaging mechanisms or on image-specific effects. Moreover, through the variation of inspection face as a within-subjects factor, it is also possible to test whether the adaptation effects are based on episodic memory effects. If this is the case, then participants will no longer show any adaptation effects, since there are no general variations of inspection face that are equal to all faces.

\section{Method}

Participants. Thirty-six undergraduate students from Freie Universität Berlin were tested individually ( 27 females, mean age 24.2 years). Participants volunteered for partial course credit.

Apparatus and Stimuli. The apparatus and stimuli were identical to those of Experiments 1 and 2.

Procedure. The experimental paradigm was the same as that in Experiment 1, with the exception that inspection face was varied as a within-subjects factor. This means that each participant inspected 9 pictorial faces (as in Experiment 1), out of which 3 were manipulated as minus 5,3 others as original, and the remaining 3 as plus 5 faces. The same variation was performed for the 9 so-called structural faces (whether face is pictorial or structural does not have an effect before the test phase runs). As in Experiment 1, the delay between the inspection phase and the test phase amounted to $5 \mathrm{~min}$. The assignment of faces to the specific experimental condition was balanced across participants.

\section{Results and Discussion}

On average, $95.6 \%$ of all target faces presented in the test phase were familiar to the participants. As in the experiments before, only these familiar faces were included in the following analyses (see mean data in Table 3).

Adaptation effects were tested by conducting a threeway repeated-measures ANOVA with the within-subjects factors inspection face (minus5, original, plus5), overlap (pictorial, structural, transfer), and start-test face (minus 1, original, plus1). Test face was used as the dependent variable. Note that the overlap condition "transfer" was not genuinely linked to any specific inspection face, since all transfer faces were new in the test phase and, in contrast to Experiments 1 and 2, inspection face was varied within participants. However, in order to be able to contrast the other overlap conditions with transfer (which represents some sort of a base rate) we randomly assigned $1 / 3$ of all transfer trials to minus 5 , original, and plus 5 .

The analysis revealed main effects of inspection face $\left[F(2,68)=13.15, p<.0001, \eta_{\mathrm{p}}^{2}=.279\right]$, with the following means: minus $5(M=-0.11)$, original $(M=0.05)$, and plus5 $(M=0.38)$. Bonferroni comparisons revealed significant differences between minus 5 and plus 5 ( $p<$ $.0001)$, original and plus5 $(p=.0019)$, but not between minus 5 and original ( $p=.0713$, n.s.). Additionally, there was a main effect of start-test face $[F(2,68)=36.71, p<$ $\left..0001, \eta_{\mathrm{p}}^{2}=.519\right]$, with the following means: minus $1(M=$ $-0.22)$, original $(M=0.12)$, and plus1 $(M=0.42)$. Bonferroni comparisons revealed significant differences between all possible pairs $(p s<.0001)$. Furthermore, there was an interaction between overlap and inspection face $\left[F(4,136)=3.18, p=.016, \eta_{\mathrm{p}}^{2}=.086\right]$ (see Figure 4). We further investigated the interaction between overlap and inspection face by calculating the simple main effects of inspection face. There were only effects of inspection face for the overlap conditions pictorial and structural $\left[F_{\mathrm{S}}(2,33)>6.53, p \mathrm{~s}<.004, \eta_{\mathrm{p}}^{2} \mathrm{~s}>.284\right]$, but not for transfer $\left[F_{\mathrm{s}}(2,33)<1\right.$, n.s. $]$ (see Figure 5).

These analyses revealed two important results. (1) The long-term adaptation effects found in Experiments 1 and 2 were not only based on the specific paradigm used there, but were also evident in a paradigm in which all variations of inspection face were used within participants. Thus, the adaptation effect could not be a simple episodic memory effect in which participants memorized general deviations toward a specific direction and based their evaluations on these tendencies. Moreover, since inspection face was varied within participants, the adaptation effects could also not be based on a simple averaging mechanism of all instances seen during the inspection phase, because then there should no longer be an effect of inspection face at all. (2) The adaptation effects were once again shown to be based on identity-specific, and thus person-based, effects, but not on image-based effects. This result is an indication for a structural (see Bruce \& Young, 1986) but not a pictorial code that is touched by the adaptation effects.

In comparison with the preceding experiments, Experiment 3 showed a deviated pattern concerning the factor inspection face. Here, the adaptation to minus 5 faces was

Table 3

Mean Selections and Standard Deviations As Veridical for Experiment 3

\begin{tabular}{|c|c|c|c|c|c|c|c|}
\hline \multirow[b]{3}{*}{ Overlap } & \multirow{3}{*}{$\begin{array}{l}\text { Start-Test } \\
\text { Face }\end{array}$} & \multicolumn{6}{|c|}{ Inspection Face } \\
\hline & & \multicolumn{2}{|c|}{ minus 5} & \multicolumn{2}{|c|}{ original } & \multicolumn{2}{|c|}{ plus 5} \\
\hline & & $M$ & $\overline{S D}$ & $M$ & $S D$ & $M$ & $S D$ \\
\hline \multirow[t]{3}{*}{ Pictorial } & minus 1 & -0.47 & 1.18 & -0.32 & 0.78 & 0.14 & 1.03 \\
\hline & original & -0.18 & 1.12 & -0.04 & 0.93 & 0.51 & 0.91 \\
\hline & plus 1 & 0.12 & 1.22 & 0.35 & 0.86 & 0.86 & 1.04 \\
\hline \multirow[t]{3}{*}{ Structural } & minus 1 & -0.55 & 0.76 & -0.25 & -0.78 & 0.21 & 1.18 \\
\hline & original & -0.25 & 1.00 & 0.13 & 0.96 & 0.51 & 1.05 \\
\hline & plus 1 & 0.13 & 1.10 & 0.20 & 0.85 & 0.86 & 1.18 \\
\hline \multirow[t]{3}{*}{ Transfer } & minus 1 & -0.28 & 0.75 & -0.34 & 0.97 & -0.13 & 1.04 \\
\hline & original & -0.07 & 1.01 & 0.25 & 0.87 & 0.19 & 0.97 \\
\hline & plus1 & 0.54 & 0.88 & 0.49 & 0.96 & 0.25 & 1.04 \\
\hline
\end{tabular}




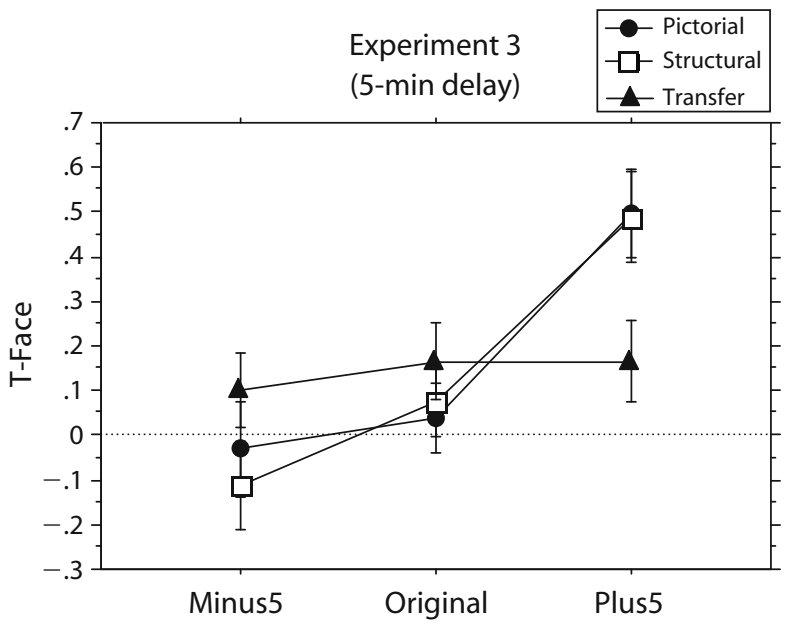

Figure 5. Mean selections as veridical for Experiment 3 as a function of inspection face and overlap (pictorial, structural, transfer). Error bars are $S E$ s.

weaker than that to plus 5 versions, indicated by a nonlinear function of inspection face in relation to the selected test face. To test whether this effect is based on irregularities in the stimulus material, we conducted a post analysis in which all face stimuli were reassessed in terms of (1) physical and (2) psychological aspects. Physically, minus 5 and plus 5 versions did not differ to the degree of alteration (pixel differences from the original ones; $m i$ nus 5, 18.03; plus5, 18.08; $p=.29$, n.s.). However, with respect to psychological aspects, using a post test in which 6 participants had to evaluate the similarity between the original and the minus 5 or the plus 5 version (simultaneous matching task; Likert scale from 1, very unsimilar to 7 , very similar), a significant difference between both extreme versions was found (minus 5, 3.89; plus5, 3.44; $\left.p=.0070, \eta_{\mathrm{p}}^{2}=.129\right)$. This small but reliable effect might be the basis of the deviation from the linear trend seen in Figure 5; another reason might be the asymmetric craniofacial distribution of both facial changes (Hreczko \& Farkas, 1994), which possibly modulated the size of the adaptation effects. The exact basis of this effect (which was also observable although much weaker in the former experiments, see Figure 4) remains speculative within the frame of the studies presented here.

\section{Reanalyses of the Selection Strategy in the Test Phase}

As was discussed previously, although all experiments showed strong evidence for adaptation effects on an identity-related basis, there was also evidence on a more general basis, as indicated by the additional effects for transfer faces in Experiments 1 and 2.

In order to better understand the underlying processes, we reanalyzed the data, especially for the experiments that varied inspection face in a between-subjects manner (Experiments 1 and 2) by integrating additional information about the process of selecting the target face in the test phase. The rationale for these analyses is based on the following findings. Since we have found reliable strong effects of start-test face, it is highly plausible that the specific movement pattern through the series of presented faces in the selection process of the test phase influenced the final selection. Concretely, if participants performed the veridicality selection process based on previously seen manipulations of the inspection faces, then not only the direct effect of inspection face would form the basis of the adaptation, but also the indirect effect of presented face versions in the test phase.

In order to test this idea, we reanalyzed the data by integrating a covariate that indicates which image each participant inspected the most frequently in the test phase as a covariate. This variable WMC (weighted mean count) was calculated by weighting more extreme face versions (e.g., minus 5, plus5, minus 4, plus4, . .) higher than faces closer to the original (e.g., minus1, original, plus1, ...). For example, if a participant started the test phase by being exposed to a minus 1 face as a start-test-face, and then changed this version to a minus 2 , minus 3 with finally selecting a minus 2 version, then this movement pattern would be calculated as a WMC of $[(-1)+(-2)+$ $(-3)+(-2) / 4=-2]$. In addition to this variable, we calculated a second covariate WMT (weighted mean, based on time), which additionally integrates temporal aspects. Thus, given the same movement pattern as above and respective inspection times of $1,000 \mathrm{msec}, 1,000 \mathrm{msec}$, $5,000 \mathrm{msec}$, and $1,000 \mathrm{msec}$, we would obtain a WMT of $[(-1 \times 1,000 \mathrm{msec})+(-2 \times 1,000 \mathrm{msec})+(-3 \times$ $5,000 \mathrm{msec})+(-2 \times 1,000 \mathrm{msec}) / 8,000 \mathrm{msec}=-2.5]$. The WMT measurement follows the idea that the longer the inspection of a specific face version, the stronger the adaptation effect will be. This rationale is based on the idea of Miller, Westerman, and Lloyd (2004), who argued that the amount of time that participants spent looking at a stimulus is an important predictor for memory recognition in general, and the primacy effect in memory for repetitions in particular.

We reanalyzed all ANOVAs described for the previous experiments separately with either the covariate $\mathrm{WMC}$ or WMT; thus, we conducted 2 (WMC or WMT) $\times 3$ (experiments) separate ANCOVAs. Both covariates demonstrated large effects on the finally selected test face in all experiments (see Table 4).

Moreover, as indicated by the statistics provided in Table 4, even though these covariates had a large impact on the selection of the test face, the factor inspection face still remained a powerful predictor for the adaptation effect. Thus, the effect of the inspection face was not an artifact of the specific test procedure, but seems to be the basis of a genuine adaptation effect based on the specific inspection of faces in the inspection phase.

We further investigated the transfer effect of Experiment 1 (5-min delay condition) and Experiment 2 (24-h delay condition) by analyzing the correlations between inspection face and selected test face when WMC/WMT was partialed out. If the effect on transfer faces is a genuine adaptation effect, then there must be reliable correlations between the inspection face and the selected test face, even after a delay of $24 \mathrm{~h}$. Concerning these partial 
Table 4

Reanalyses of the Data by ANCOVAs With Either the Covariate WMC (Weighted Mean Count) or WMT (Weighted Mean, Based on Time)

\begin{tabular}{|c|c|c|c|c|}
\hline \multirow[b]{2}{*}{ Experiment } & \multicolumn{2}{|c|}{ WMC } & \multicolumn{2}{|c|}{ WMT } \\
\hline & Inspection Face & Covariate & Inspection Face & Covariate \\
\hline 1 & $p=.004, \eta_{\mathrm{p}}^{2}=.294$ & $p<.0001, \eta_{\mathrm{p}}^{2}=.565$ & $p=.001, \eta_{\mathrm{p}}^{2}=.366$ & $p<.0001, \eta_{\mathrm{p}}^{2}=.528$ \\
\hline 2 & $p=.043, \eta_{\mathrm{p}}^{2}=.178$ & $p<.0001, \eta_{\mathrm{p}}^{2}=.828$ & $p=.025, \eta_{\mathrm{p}}^{2}=.206$ & $p<.0001, \eta_{\mathrm{p}}^{2}=.893$ \\
\hline 3 & $p<.0001, \eta_{\mathrm{p}}^{2}=.253$ & $p<.0001, \eta_{\mathrm{p}}^{2}=.413$ & $p<.0001, \eta_{\mathrm{p}}^{2}=.263$ & $p<.0001, \eta_{\mathrm{p}}^{2}=.482$ \\
\hline
\end{tabular}

Note-Because of a better readability, only significant effects (or near-to-dignificant rffects) of the main factor of inspection face and main effects of WMC and WMT are reported (all experiments).

correlations, we obtained very similar results to the aforementioned ANCOVAs. Again, there were reliable correlations for all overlap conditions (Table 5) in Experiment 1, which used a delay of $5 \mathrm{~min}$.

However, in Experiment 2, which used a delay of $24 \mathrm{~h}$, there was no correlation for the transfer overlap condition between inspection face and the selected test face when WMC/WMT was partialed out. This result indicates that the transfer effects obtained for Experiment 1 were rather artificial, probably induced by the specific procedure of varying inspection face between participants.

\section{GENERAL DISCUSSION}

The results of the present three experiments show that veridicality decisions relating to highly familiar faces were systematically deviated toward previously inspected distorted face versions. These adaptation effects were effective after $5 \mathrm{~min}$ (Experiments 1 and 3 ) and even after $24 \mathrm{~h}$ (Experiment 2). Moreover, we demonstrated that such adaptations are not only limited to the same pictorial version of one's identity, but that they can also be observed for alternative images of one's face (shown by similar effects for pictorial and structural faces). Thus, such adaptations seem to work on a more structural than pictorial processing level (Bruce \& Young, 1986). Importantly, the adaptation effects obtained after $24 \mathrm{~h}$ were weakened compared with those effects obtained just after 5 min, but they were still reliable. Although these experiments also showed that there were strong transfer effects to faces that had not been inspected in the inspection phase, reanalyses indicated that obtained transfer effects were primarily caused by the specific procedure used in Experiments 1 and 2.

Table 5

Partial Correlations Between Inspection Face and Selected Test Face for the Overlap Conditions Pictorial, Structural, and Transfer When the WMC (Weighted Mean Count) or WMT (Weighted Mean, Based on Time) Was Partialed Out (Experiments 1 and 2)

\begin{tabular}{clll}
\hline Experiment & $\begin{array}{c}\text { Overlap } \\
\text { Condition }\end{array}$ & \multicolumn{1}{c}{$\begin{array}{c}\text { WMC } \\
\text { Partialed Out }\end{array}$} & \multicolumn{1}{c}{$\begin{array}{c}\text { WMT } \\
\text { Partialed Out }\end{array}$} \\
\hline \multirow{2}{*}{1} & Pictorial & $r=.621, p<.0001$ & $r=.646, p<.0001$ \\
& Structural & $r=.653, p<.0001$ & $r=.689, p<.0001$ \\
& Transfer & $r=.427, p=.010$ & $r=.551, p=.001$ \\
2 & Pictorial & $r=.475, p=.004$ & $r=.429, p=.010$ \\
& Structural & $r=.602, p=.008$ & $r=.429, p=.010$ \\
& Transfer & $r=.071$, n.s. & $r=.014$, n.s. \\
\hline
\end{tabular}

\section{Flexibility of Familiar Faces}

The present experiments showed that face adaptation effects that were already demonstrated for unfamiliar faces (see, e.g., Webster \& MacLin, 1999) are also expandable to highly familiar faces (see Carbon \& Leder, 2005a, 2006a). Judgments concerning the veridicality of highly familiar faces, whose representations are thought to be highly reliable (Bruce et al., 2001; Carbon \& Leder, 2005 b), were strongly influenced by recent visual inputs.

However, does such a proposition not run counter to nearly every current theory of face recognition? Most theories of face recognition seek to understand or discover the nature of the representations that allow face recognition to be sufficiently stable, despite variations in the input. In the case of our experiments, participants were able to identify the celebrities for every face version. Thus, the adaptation effect was not so profound that identification failed. What did fail, however, was the capability of identifying the veridical version of a face. Furthermore, the participants did not wrongly select extreme versions (which had been inspected previously) as veridical versions; rather, they selected slightly deviated versions toward these extreme images. Or, in other words: The perception of these faces adapted toward the experimentally inspected versions. Such a mechanism that flexibly adapts face perception seems to be important for everyday life, since faces change slowly and often in rather subtle ways over time. It is essential for a perceptual system to be "up to date" with all visual information available at the time. Very distinctive information - for example, the extreme versions of faces (the inspected minus 5 or plus 5) used here-seems to be particularly important input for such a mechanism. This explanation is in accordance with the idea that familiar stimuli or stimuli that are not very distinctive are given less attention than novel stimuli or very distinctive stimuli (DiGirolamo \& Hintzman, 1997; see also Miller et al., 2004). Essentially, the following "cognitive balance" has to be fulfilled: Representations must be stable enough to enable reliable identification, but the perceptual system must, on the other hand, also be sufficiently flexible to adapt toward new information. Asking participants to judge the veridicality of a face, as we have done in our experiments, appears to be a suitable and very sensitive task for analyzing such adaptation effects.

\section{Duration of the Effect}

An important question about the adaptation effect is how long the effect lasts (see Leopold et al., 2005). Ex- 
periments 1 and 3 demonstrated that adaptation effects were still present after a delay of 5 min between inspection and test phase; Experiment 2 even showed that adaptation effects could be demonstrated after a delay of $24 \mathrm{~h}$. Thus, the suggestion is that such adaptation effects are not only based on simple iconic traces or simple visual aftereffects. It is a question of systematic future research to determine whether these changes are temporary or permanent, and whether they are based on perceptual or representational grounds. In any case, we assume that after having seen several veridical versions of the deviated faces postexperimentally, the effect will vanish again. Such a "resetting" or "readjustment" mechanism would be in line with the adaptation effect itself. However, we also believe that very distinctive versions of a face, for instance, those presently realized by inspected minus5/plus 5 versions, are much more effective for adaptation than relatively indistinctive versions - provided that such distinctive versions are still recognized as being associated with the respective face.

\section{Specificity of the Effect}

Varying the overlap of pictorial information between pictures in the inspection and test phase allows us to dissociate pictorial-specific effects, image-specific effects, and more general effects. All experiments demonstrated that the adaptation effects are not only based on simple pictorial grounds, but are also based on broader concepts, such as structural codes. Thus, it made no difference whether inspected face versions were identical between the inspection and test phases. This result extends previously found structural effects demonstrated by a partial impenetrability of adaptation effects - for example, where size, position, and angle between inspection and test did not change the efficiency of adaptation (Leopold et al., 2001).

Moreover, in Experiments 1 and 2, adaptation effects were even observable (although weaker) for faces that had not been inspected in the inspection phase at all. Covariance analyses showed that this transfer effect was probably based on artificial effects due to the specific test procedure used here, especially to the 24 -h delay between inspection and test phase in Experiment 2. This evidence was further fueled by the results of Experiment 3, which used an altered experimental design to address this question. In conclusion, adaptation effects found in the present study are mainly based on structural coding that is identity specific and not only image specific.

\section{Recollection or Familiarity?}

Current memory theories propose that recognition memory is supported by two different memory processes - that is, explicit and implicit memory. The former is often labeled as recollection, whereas the latter is often labeled as recognition of familiarity (Duarte, Ranganath, Winward, Hayward, \& Knight, 2004; Mandler, 1980; Tulving, 1985). Both types of memory are supposed to rely on distinct memory systems (Drosopoulos, Wagner, \& Born, 2005; Jacoby, Toth, \& Yonelinas, 1993). If we assume that the adaptation effects found here were caused by a kind of priming mechanism, then the observed effects would not be based on recollection. Priming in this respect would mean that inspected faces primed and therefore facilitated the processing of highly similar faces. In this case, the adaptation effects would be based on the feeling of familiarity (Rajaram \& Geraci, 2000). However, three facts appear to be problematic for this account. (1) The inspected versions minus 5 or plus 5 were very different from the slightly altered faces, which were selected later in the test phase. Thus, the proposed priming mechanism must work quite structurally. (2) In all experiments, not only versions that were based on the same images (pictorial overlap condition), but also those that were based on alternative images (structural overlap condition) were used in the experimental paradigms. However, the adaptation effects for this condition were equally strong as those in the pictorial-identical condition. (3) Experiment 3 used a within-subjects variation of inspection face with faces of 27 different celebrities; thus, faces of 9 celebrities were manipulated minus 5 versions, 9 were original versions, and the rest were plus 5 versions. If the adaptation effect was based on familiarity (or processing fluency; Westerman, Lloyd, \& Miller, 2002) in the sense of recognition without contextual knowledge, it seems implausible that the inspection face would still have a systematic effect on the selection of the test face. This interpretation is also in accordance with the idea that the adaptation effects revealed here are not subject to episodic memory effects (Tulving, 1972), in which participants are supposed to remember the episode of a general variation of inspection face.

\section{Conclusion}

We have documented the adaptation effect, which rapidly biases the perception of highly familiar faces toward previously inspected faces. This effect does not seem to be based on a simple pictorial adaptation mechanism; rather, it seems to work according to a quite structural one, as indicated by adaptation effects transferring to alternative image versions of a familiar face. Moreover, the adaptation lasts for an extended period of one day after the experiment. Equipped with such an adaptation ability, the cognitive system is flexible to adapt to a constantly changing environment by integrating the given information as a relevant source. Such an efficient and automatic adaptation might constitute the illusion of having a stable perception, whilst this perception is in fact being permanently adapted, tuned, and optimized.

\section{AUTHOR NOTE}

We thank Thomas Ditye and Martin Schwarz for their assistance in collecting the data and Ruth Mainka and Andrea Lyman for proofreading the text. We also thank three anonymous reviewers and James Bartlett for their very helpful comments on earlier versions of this article, and an anonymous reviewer who inspired us to conduct the three experiments presented in this article. Please address all correspondence to C.-C. Carbon, University of Vienna, Faculty of Psychology, A-1010 Vienna, Austria (e-mail: ccc@experimental-psychology.com).

\section{REFERENCES}

Baddeley, A. D. (1998). Human memory: Theory and practice (rev. ed.). Needham Heights, MA: Allyn and Bacon.

Benson, P. J., \& Perrett, D. I. (1993). Extracting prototypical facial images from exemplars. Perception, 22, 257-262. 
Benson, P. J., \& Perrett, D. I. (1994). Visual processing of facial distinctiveness. Perception, 23, 75-93.

Bower, G. H., Thompson-Schill, S., \& Tulving, E. (1994). Reducing retroactive interference: An interference analysis. Journal of Experimental Psychology: Learning, Memory, \& Cognition, 20, 51-66.

BRUCE, V. (1994). Stability from variation: The case of face recognition. Quarterly Journal of Experimental Psychology, 47A, 5-28.

Bruce, V., Doyle, T., Dench, N., \& Burton, M. (1991). Remembering facial configurations. Cognition, 38, 109-144.

Bruce, V., Henderson, Z., Newman, C., \& Burton, A. M. (2001). Matching identities of familiar and unfamiliar faces caught on CCTV images. Journal of Experimental Psychology: Applied, 7, 207-218.

BRuce, V., \& Young, A. (1986). Understanding face recognition. British Journal of Psychology, 77, 305-327.

CARbon, C.-C., \& Leder, H. (2005a). Face adaptation: Changing stable representations of familiar faces within minutes? Advances in Cognitive Psychology, 1, 1-7.

CARbon, C.-C., \& Leder, H. (2005b). When feature information comes first! Early processing of inverted faces. Perception, 34, 1117-1134.

Carbon, C.-C., \& Leder, H. (2006a). The Mona Lisa effect: Is “our" Lisa fame or fake? Perception, 35, 411-414.

Carbon, C.-C., \& Leder, H. (2006b). When faces are heads: Viewdependent recognition of faces altered relationally or componentially. Swiss Journal of Psychology, 65, 245-252.

Cohen, J. D., MacWhinney, B., Flatt, M., \& Provost, J. (1993). PsyScope: A new graphic interactive environment for designing psychology experiments. Behavior Research Methods, Instruments, \& Computers, 25, 257-271.

Collishaw, S. M., \& Hole, G. J. (2000). Featural and configurational processes in the recognition of faces of different familiarity. Perception, 29, 893-909.

DiGirolamo, G. J., \& Hintzman, D. L. (1997). First impressions are lasting impressions: A primacy effect in memory for repetitions. Psychonomic Bulletin \& Review, 4, 121-124.

Drosopoulos, S., Wagner, U., \& Born, J. (2005). Sleep enhances explicit recollection in recognition memory. Learning \& Memory, 12 , 44-51.

Duarte, A., Ranganath, C., Winward, L., Hayward, D., \& Knight, R. T. (2004). Dissociable neural correlates for familiarity and recollection during the encoding and retrieval of pictures. Cognitive Brain Research, 18, 255-272.

Faulkner, T. F., Rhodes, G., Palermo, R., Pellicano, E., \& FerGUSON, D. (2002). Recognizing the un-real McCoy: Priming and the modularity of face recognition. Psychonomic Bulletin \& Review, 9, $327-334$

Goldstein, A. G., \& Chance, J. E. (1980). Memory for faces and schema theory. Journal of Psychology, 105, 47-59.

HreczKo, T. A., \& Farkas, L. G. (1994). Norms of the craniofacial asymmetries in North American Caucasians. In L. G. Farkas (Ed.), Anthropometry of the head and face (2nd ed., pp. 359-380). New York: Raven.

Jacoby, L. L., Toth, J. P., \& Yonelinas, A. P. (1993). Separating conscious and unconscious influences of memory: Measuring recollection. Journal of Experimental Psychology: General, 122, 139-154.

KöHLER, W., \& WaLLaCH, H. (1944). Figural aftereffects: An investigation of visual processes. Proceedings of the American Philosophical Society, 88, 269-357.

LEDER, H., \& BRUCE, V. (2000). When inverted faces are recognized: The role of configural information in face recognition. Quarterly Journal of Experimental Psychology, 53A, 513-536.

Leder, H., \& CARBON, C.-C. (2005). When context hinders! Learn-test compatibility in face recognition. Quarterly Journal of Experimental Psychology, 58A, 235-250.

LedER, H., \& CARBON, C.-C. (2006). Face-specific configural processing of relational information. British Journal of Psychology, 97, 19-29.

Lee, K., Byatt, G., \& Rhodes, G. (2000). Caricature effects, distinctiveness, and identification: Testing the face-space framework. Psychological Science, 11, 379-385.
Leopold, D. A., O’Toole, A. J., Vetter, T., \& Blanz, V. (2001). Prototype-referenced shape encoding revealed by high-level aftereffects. Nature Neuroscience, 4, 89-94.

Leopold, D. A., Rhodes, G., MÜller, K.-M., \& JefFery, L. (2005). The dynamics of visual adaptation to faces. Proceedings of the Royal Society of London B, 272, 897-904.

MandLeR, G. (1980). Recognizing: The judgment of previous occurrence. Psychological Review, 87, 252-271.

Miller, J. K., Westerman, D. L., \& Lloyd, M. E. (2004). Are first impressions lasting impressions? An exploration of the generality of the primacy effect in memory for repetitions. Memory \& Cognition, 32, 1305-1315.

Mondloch, C. J., Le Grand, R., \& Maurer, D. (2002). Configural face processing develops more slowly than featural face processing. Perception, 31, 553-566.

Rajaram, S., \& Geraci, L. (2000). Conceptual fluency selectively influences knowing. Journal of Experimental Psychology: Learning, Memory, \& Cognition, 26, 1070-1074.

Rhodes, G., JefFery, L., Watson, T. L., Jaquet, E., Winkler, C., \& ClifFord, C. W. (2004). Orientation-contingent face aftereffects and implications for face-coding mechanisms. Current Biology, 14, 2119 2123.

Rossion, B., Schiltz, C., Robaye, L., Pirenne, D., \& Crommelinck, M. (2001). How does the brain discriminate familiar and unfamiliar faces? A PET study of face categorical perception. Journal of Cognitive Neuroscience, 13, 1019-1034

Schwaninger, A., Carbon, C.-C., \& Leder, H. (2003). Expert face processing: Specialization and constraints. In G. Schwarzer \& H. Leder (Eds.), The development of face processing (pp. 81-97). Göttingen: Hogrefe \& Huber

Tulving, E. (1972). Episodic and semantic memory. In E. Tulving \& W. Donaldson (Eds.), Organization of memory (pp. 381-403). New York: Academic Press.

Tulving, E. (1985). How many memory systems are there? American Psychologist, 40, 385-398.

Valentine, T., \& Bruce, V. (1986a). The effects of distinctiveness in recognising and classifying faces. Perception, 15, 525-535.

VAlentine, T., \& BRUCE, V. (1986b). Recognizing familiar faces: The role of distinctiveness and familiarity. Canadian Journal of Psychology, 40, 300-305.

Webster, M. A., Kaping, D., Mizokami, Y., \& Duhamel, P. (2004). Adaptation to natural facial categories. Nature, 428, 557-561.

Webster, M. A., \& MacLin, O. H. (1999). Figural aftereffects in the perception of faces. Psychonomic Bulletin \& Review, 6, 647-653.

Westerman, D. L., Lloyd, M. E., \& Miller, J. K. (2002). The attribution of perceptual fluency in recognition memory: The role of expectation. Journal of Memory \& Language, 47, 607-617.

\section{NOTES}

1. Face adaptation effects are sometimes also termed figural aftereffects (see, e.g., Webster \& MacLin, 1999). The figural aftereffect (Köhler \& Wallach, 1944) is an aftereffect caused by prolonged inspection of an encompassing figure - for example, a circle - which gives rise to certain aftereffects whose characteristics are revealed as apparent distortions in test figures that are subsequently inspected.

2. Franz Beckenbauer and Rudi Völler (soccer legends and sport representatives), Pierce Brosnan, Nicolas Cage, George Clooney, Tom Cruise, Pamela Anderson, Cameron Diaz, Nicole Kidman, Jack Nicholson, Brad Pitt, Julia Roberts (actors), George W. Bush, Bill Clinton, Angela Merkel, Gerhard Schröder, Helmut Kohl (politicians), Cindy Crawford, Claudia Schiffer (models), Princess Diana (royalty), Verona Feldbusch, Thomas Gottschalk (German TV stars), Oliver Kahn, Michael Schumacher, and Boris Becker (athletes), Madonna (singer), Arnold Schwarzenegger (actor/politician).

(Manuscript received January 1, 2006; revision accepted for publication February 19, 2007.) 